\title{
EFICIENCIA DE SUELO Y TEZONTLE EN SISTEMAS DE PRODUCCIÓN DE TOMATE EN INVERNADERO
}

\author{
EFFICIENCY OF SOIL AND TEZONTLE IN PRODUCTION SYSTEMS OF TOMATO \\ UNDER GREENHOUSE
}

\author{
José L. Ojodeagua Arredondo ${ }^{1}$, Javier Z. Castellanos Ramos ${ }^{1 *}$, J. Jesús Muñoz Ramos ${ }^{1}$, Gabriel Alcántar \\ González ${ }^{2}$, Leonardo Tijerina Chávez ${ }^{3}$, Patricia Vargas Tapia ${ }^{1}$ y Sergio Enríquez Reyes ${ }^{1}$
}

\begin{abstract}
${ }^{1}$ Unidad de Horticultura Protegida, Campo Experimental Bajío, Instituto Nacional de Investigaciones Forestales, Agrícolas y Pecuarias. Km 6.5 Carr. Celaya-San Miguel de Allende. 38110, Celaya, Guanajuato, México. ${ }^{2}$ Programas de Edafología e ${ }^{3}$ Hidrociencias, Colegio de Postgraduados-Campus Montecillo. 56230, Texcoco, Edo. de México, México.
\end{abstract}

*Autor para correspondencia (javier.castellanos.ramos@gmail.com)

\section{RESUMEN}

En México $80 \%$ de la producción hortícola en invernadero se lleva a cabo en suelo y en la restante se utiliza algún tipo de sustrato inerte. En este estudio se determinó el rendimiento comercial, la eficiencia en el uso del agua (EUA) y de nutrimentos del cultivo de tomate (Lycopersicon esculentum Mill. cv. 'Gironda') en dos sustratos, suelo y tezontle. El rendimiento comercial en suelo fue de $34.1 \mathrm{~kg}$ $\mathrm{m}^{-2}$ con un suministro nutrimental total aplicado de $920,342,1520$, 1050 y $290 \mathrm{~kg} \mathrm{ha}^{-1}$ de N, $\mathrm{P}_{2} \mathrm{O}_{5}, \mathrm{~K}_{2} \mathrm{O}$, Ca y $\mathrm{Mg}$ respectivamente, y una eficiencia en el uso de nutrimentos de 2.7, 1.0, 4.5, 3.1 y $0.9 \mathrm{~kg} \mathrm{t}^{-1} \mathrm{de}$ fruta comercial de los mismos nutrimentos, en un suelo que no había sido usado previamente para la horticultura. El suministro hídrico total a este tratamiento fue de $801 \mathrm{~L} \mathrm{~m}^{-2}$, con una EUA de $43 \mathrm{~kg}$ de fruta $\mathrm{m}^{-3}$ de agua. Los rendimientos comerciales en los tratamientos de tezontle fueron estadísticamente iguales, con un promedio de 31.4 $\mathrm{kg} \mathrm{m}^{-2}$, un suministro nutrimental promedio total de 1748, 452, 3636,2170 y $731 \mathrm{~kg} \mathrm{ha}^{-1}$ de $\mathrm{N}, \mathrm{P}_{2} \mathrm{O}_{5}, \mathrm{~K}_{2} \mathrm{O}$, Ca y $\mathrm{Mg}$, y una eficiencia promedio en el uso de nutrimentos de 5.6, 1.4, 11.6, 6.9 y $2.3 \mathrm{~kg} \mathrm{t}^{-1}$ de fruta, respectivamente. Las pérdidas de nutrimentos en el drenaje de los tratamientos de tezontle fueron, en promedio, de 56, 6, 42, 48 y $60 \%$ respectivamente, con un suministro hídrico total de 1114 $\mathrm{L} \mathrm{m}^{-2}$, un drenaje de $36 \%$ y una EUA de $28.2 \mathrm{~kg} \mathrm{~m}^{-3}$. Estos resultados indican el potencial del suelo en el ahorro de agua y fertilizante, ya que la productividad en ambos sistemas fue igual.

Palabras clave: Lycopersicon esculentum, sustrato, uso eficiente de agua, uso eficiente de fertilizante.

\section{SUMMARY}

In México, $80 \%$ of the greenhouse area is cultivated under soil conditions and in the remaining some type of inert substrate is used. In this study we determined the commercial yield, water and nutrient use efficiency of tomato (Lycopersicon esculentum Mill. cv. 'Gironda') grown in two substrates, soil and tezontle. Commercial yield under soil conditions was $34.1 \mathrm{~kg} \mathrm{~m}^{-2}$, with a total nutrient supply of $920,342,1520,1050$ y $290 \mathrm{~kg} \mathrm{ha}^{-1}$ of $\mathrm{N}, \mathrm{P}_{2} \mathrm{O}_{5}, \mathrm{~K}_{2} \mathrm{O}$, Ca y $\mathrm{Mg}$, respectively, and a nutrient use efficiency of $2.7,1.0,4.5,3.1$ y $0.9 \mathrm{~kg}$ $\mathrm{t}^{-1}$ of commercial fruit for those nutrients, in a soil not previously used for horticulture. Total water supply was $801 \mathrm{~L} \mathrm{~m}^{-2}$, with a water use efficiency (WUE) of $43 \mathrm{~kg}$ of fruit $\mathrm{m}^{-3}$ of water. Commercial yields of tomato in the tezontle treatments were statistically equal whit an average of $31.4 \mathrm{~kg} \mathrm{~m}^{-2}$, and an average nutrient supply of $1748,452,3636,2170$ y $731 \mathrm{~kg} \mathrm{ha}^{-1}$ of $\mathrm{N}, \mathrm{P}_{2} \mathrm{O}_{5}, \mathrm{~K}_{2} \mathrm{O}$, Ca y $\mathrm{Mg}$ respectively, and an average nutrient use efficiency of 5.6, 1.4, 11.6,

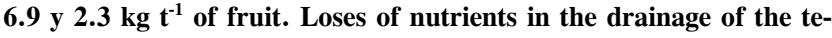
zontle treatments averaged 56, 6, 42, 48 y $60 \%$ respectively, with a total water supply of $1114 \mathrm{~L} \mathrm{~m}^{-2}$, a drainage of $36 \%$ and an WUE of $28.2 \mathrm{~kg} \mathrm{~m}^{-3}$. These results show the potential of soil for saving water and nutrients, since the productivity in both systems was the same.

Index words: Lycopersicon esculentum, substrate, water use efficiency, fertilizer use efficiency.

\section{INTRODUCCIÓN}

La industria mexicana de horticultura protegida se ha venido desarrollando en muchas regiones y en condiciones heterogéneas de clima, suelo y calidad de agua (Muñoz, 2003). Se estima que $80 \%$ de la producción hortícola bajo cubiertas plásticas se lleva a cabo en suelo (Castellanos, 2004) y la restante en algún tipo de sustrato inerte. Las ventajas del cultivo en suelo son: amortigua interrupciones temporales de agua y nutrimentos, sin afectar seriamente su desarrollo, e incrementa la eficiencia en el uso de nutrimentos y agua (Villareal et al., 2002), comparado con el uso de sustrato que requiere de 20 a $30 \%$ más de riego para evitar acumulación de sales en el medio de cultivo (Papadopoulos, 1991), lo que significa pérdida de agua y nutrimentos.

Una desventaja importante del cultivo en suelo es la presencia de enfermedades, debido a que el agricultor repite año tras año los mismos cultivos en el mismo terreno. Actualmente, hay un renovado interés en la producción en 
suelo y tiene que ver con el reciente surgimiento de la técnica de injerto en hortalizas que se está imponiendo en el mundo bajo condiciones protegidas, como una estrategia para enfrentar las enfermedades provenientes del suelo (Camacho-Ferre y Tello-Marquina, 2006); así, las enfermedades pasan a ser un problema de menor relevancia y el cultivo en suelo es una posibilidad atractiva.

Estudios realizados con el uso de fertirrigación muestran resultados de producción de tomate (Lycopersicom esculentum Mill.) a campo abierto de 59 a $61.2 \mathrm{t} \mathrm{ha}^{-1}$ (Alcántar y Villareal, 1999; Villareal et al., 2002), mientras que en invernadero la producción es de 192 a $262 \mathrm{t} \mathrm{ha}^{-1}$ (Grijalva-Contreras et al., 2004) de frutos comerciales, si las condiciones de fertilidad y fitosanidad del suelo lo permiten.

El uso de sustratos inertes se ha venido implantando en la producción de tomate en invernadero, con rendimientos hasta de $330 \mathrm{t} \mathrm{ha}^{-1}$ cuando se utiliza lana de roca como sustrato (Hao y Papadoupulos, 2002), pero también hay pérdidas importantes de agua y nutrimentos por efecto del drenaje (Papadopoulos, 1991). El uso de tezontle como medio de cultivo se ha estudiado en la producción de hortalizas (Baca et al., 1991) desde hace unos 15 años. El tezontle es un material considerado como inerte desde el punto de vista químico, cuyo extracto de saturación tiene un $\mathrm{pH}$ próximo a la neutralidad, su capacidad de intercambio catiónico es muy baja, buena aireación, retención de humedad que varía con el diámetro de las partículas, generalmente está libre de sustancias tóxicas y tiene buena estabilidad física (Bastida, 1999), además de su bajo costo de adquisición (Castellanos y Vargas-Tapia, 2003). Por ello ha crecido el interés en comparar diferentes sistemas y sustratos para la producción de tomate en invernadero, en cuanto a rendimiento y optimización en el uso del agua y nutrimentos (Inden y Torres, 2004).

El seguimiento de la nutrición del cultivo en invernadero es importante para maximizar los rendimientos, ya sea que se cultive en suelo o en sustrato. El uso de herramientas de diagnóstico nutrimental, como el análisis de extracto celular de peciolo (ECP) (Coltman y Riede, 1992; Hochmuth, 1994), el análisis de tejido vegetal (Jones, 1999; Andriolo et al., 2002), la determinación de nutrimentos en la solución de suelo (Castellanos, 2004), así como la concentración de nutrimentos en solución nutritiva de suministro y drenaje en el caso del tezontle, y el manejo hídrico, son estrategias fundamentales para maximizar rendimientos. El objetivo del estudio fue comparar dos sistemas de producción de tomate en invernadero, suelo y sustrato, en relación a la nutrición del cultivo, rendimiento comercial y a la eficiencia de uso del agua y de fertilizantes, para elegir al más conveniente.

\section{MATERIALES Y MÉTODOS}

El estudio se hizo en instalaciones localizadas en Celaya, Guanajuato. La evaluación agronómica se llevó a cabo entre el 21 de agosto de 2002 y el 5 de mayo de 2003, con una duración de 257 d, en un invernadero tipo túnel con cubierta de polietileno. Se mantuvo una temperatura mínima nocturna por arriba de $12{ }^{\circ} \mathrm{C}$, mediante calefacción. Las temperaturas medias máxima y mínima dentro del invernadero fueron de 29 y $14.8{ }^{\circ} \mathrm{C}$, respectivamente. Se cultivó tomate variedad 'Gironda', de crecimiento vigoroso, con frutos uniformes de tipo redondo (peso 180-200 g).

Se evaluaron dos sustratos de producción hortícola: suelo y tezontle con diferente granulometría. El suelo no había sido usado previamente para la horticultura, es de textura franca con $49 \%$ de arena, $20 \%$ de arcilla y $30 \%$ limo, pH 7.6 (suelo-agua de 1:2), materia orgánica 1.18 $\%$ según el método Walkley y Black (Jackson, 1964), conductividad eléctrica del extracto de saturación de 1.2 $\mathrm{dS} \mathrm{m}{ }^{-1}$, y con $821,4100,576$ y $458 \mathrm{mg} \mathrm{kg}^{-1}$ de $\mathrm{K}, \mathrm{Ca}$, $\mathrm{Mg}$ y Na extractables respectivamente, y $11 \mathrm{mg} \mathrm{kg}^{-1} \mathrm{de} \mathrm{N}$ $\mathrm{NO}_{3}$. Se usó tezontle de color negro y rojo; el negro se cribó para obtener dos tamaños de partículas: grueso (G: $>2 \mathrm{y}<12.5 \mathrm{~mm}$ ) y fino $\mathrm{F}:<2 \mathrm{~mm}$ ), de los cuales se tomaron las siguientes proporciones (en volumen) para su mezcla y uso: a) $25 \%$ fino más $75 \%$ grueso (tratamiento TN 25/75) y b) $75 \%$ fino más $25 \%$ grueso (tratamiento TN 75/25). El rojo (tratamiento TR) se cribó solamente para eliminar los tamaños de partículas grandes $(>20 \mathrm{~mm}$ de diámetro). En el Cuadro 1 se presenta la caracterización física de cada tratamiento de tezontle.

Cuadro 1. Caracterización física de los tratamientos de tezontle empleados durante el ciclo del tomate.

\begin{tabular}{lcccccc}
\hline Tratamientos & $\begin{array}{c}\text { Densidad aparente } \\
\left(\mathrm{g} \mathrm{cm}^{-3}\right)\end{array}$ & $\begin{array}{c}\text { Densidad real } \\
\left(\mathrm{g} \mathrm{cm}^{-3}\right)\end{array}$ & $\begin{array}{c}\text { Porosidad de } \\
\text { aireación }(\%)\end{array}$ & $\begin{array}{c}\text { Capacidad de retención } \\
\text { de agua }(\%)\end{array}$ & $\begin{array}{c}\text { Espacio poroso } \\
\text { total }(\%)\end{array}$ & $\begin{array}{c}\text { Índice de grosor } \\
(\%)\end{array}$ \\
\hline${ }^{\dagger}$ TN F25/G75 & 0.61 & 2.45 & 39.2 & 27.9 & 74.7 & 81 \\
TN F75/G25 & 0.75 & 2.18 & 17.4 & 43.9 & 55.6 & 57 \\
TR & 1.33 & 2.80 & 19.9 & 35.0 & 52.6 & 77 \\
Valor sugerido ${ }^{\dagger \dagger}$ & $<0.80$ & ---- & $30-50$ & $30-40$ & $>65$ & -- \\
\hline
\end{tabular}

${ }^{\dagger} \mathrm{T}=$ Tezontle; $\mathrm{N}=$ Negro; $\mathrm{F}=$ Fino; $\mathrm{G}=$ Grueso; $\mathrm{R}=$ Rojo.

${ }^{\dagger}$ Castellanos J Z y P Vargas-Tapia (2007) Investigadores del área de horticultura protegida y sustratos del INFAP. Comunicación personal. 
El volumen de sustrato fue de $15 \mathrm{~L}$ y se estableció una planta por maceta, con una densidad de 2.7 plantas $\mathrm{m}^{-2}$, tanto en sustrato como en suelo. Se dispuso de una instalación de riego localizado, automatizado para la inyección de riego y fertilizantes. En tezontle, cada planta fue regada con un gotero antidrenante y autocompensado de $4 \mathrm{~L}$ $\mathrm{h}^{-1}$ de descarga unitaria. En suelo se utilizó cinta de riego con un gasto de $3.28 \mathrm{~L} \mathrm{~h}^{-1} \mathrm{~m}^{-1}$. La dotación de riego en suelo se hizo con una tensión de humedad entre 15 y 20 $\mathrm{kPa}$ a lo largo de la estación, mediante el uso de tensiómetros. En tezontle la gestión del riego se hizo mediante riego a tiempos, que consiste en establecer un calendario de riego con una frecuencia definida para todo un día (8 a 15 riegos por día, de 3 min cada uno), que se modificó con base en los drenajes colectados en las bandejas. La demanda de agua se abasteció mediante balance diario de suministro y drenaje, para conseguir un drenaje cercano a $30 \%$ en un día. La solución nutritiva aplicada al tratamiento de sustrato se diseñó para aplicar: $11,0.8,2.5$, 4.5, 7 y 2.0 mmoles $\mathrm{L}^{-1}$ de $\mathrm{NO}_{3}^{-}, \mathrm{H}_{2} \mathrm{PO}_{4}{ }^{-}, \mathrm{SO}_{4}{ }^{2-}, \mathrm{Ca}^{2+}, \mathrm{K}^{+}$ y $\mathrm{Mg}^{2+}$, respectivamente, y una CE de 2.5 a $2.8 \mathrm{dS} \mathrm{m}^{-1}$. La concentración de los micronutrimentos fue de: 1.0, $0.5,0.3,0.5,0.1$ y $0.05 \mathrm{mg} \mathrm{L}^{-1}$ de Fe, Mn, $\mathrm{Zn}, \mathrm{B}, \mathrm{Cu}$ y Mo, en el mismo orden. El pH de la solución se ajustó entre 5.6 y 6.2 con $\mathrm{H}_{2} \mathrm{SO}_{4}$. Para el tratamiento en suelo, la concentración promedio aplicada en la solución nutritiva fue: $8,0.6,3.2,3.8$ y $1.5 \mathrm{mmol} \mathrm{L}^{-1}{\mathrm{de} \mathrm{NO}_{3}}^{-}, \mathrm{H}_{2} \mathrm{PO}_{4}^{-}$, $\mathrm{Ca}^{2+}, \mathrm{K}^{+}$y $\mathrm{Mg}^{2+}$, respectivamente, con una CE $2.0 \mathrm{dS}$ $\mathrm{m}^{-1}$ y la misma concentración de microelementos que en el sustrato.

El agua de riego tenía $0.42,0.8,0.7,5.2,1.62,0.65$ y $6.37 \mathrm{mmol} \mathrm{L}{ }^{-1}$ de $\mathrm{K}^{+}, \mathrm{Ca}^{2+}, \mathrm{Mg}^{2+}, \mathrm{Na}^{+}, \mathrm{Cl}^{-}, \mathrm{SO}_{4}{ }^{2-}$, $\mathrm{HCO}_{3}{ }^{-}$respectivamente, $\mathrm{pH} 8.0$ y $\mathrm{CE}$ de $0.78 \mathrm{dS} \mathrm{m}^{-1}$. La solución nutritiva se preparó a partir de $\mathrm{Ca}\left(\mathrm{NO}_{3}\right)_{2} 4 \mathrm{H}_{2} \mathrm{O}$, $\mathrm{KNO}_{3}, \mathrm{~K}_{2} \mathrm{SO}_{4}, \mathrm{MgSO}_{4} 7 \mathrm{H}_{2} \mathrm{O}$ y $\mathrm{KH}_{2} \mathrm{PO}_{4}$, más micronutrimentos (complejo quelatado de microelementos, $\mathrm{Fe}$ EDTA y $\mathrm{H}_{3} \mathrm{BO}_{3}$ ). A los $182 \mathrm{~d}$ después del transplante (ddt) se eliminó el punto de crecimiento apical de las plantas (descabezado) en el racimo 18 y se aclareó a cuatro frutos por racimo. El diseño experimental fue en bloques completos con tratamientos aleatorizados con cuatro repeticiones, en grupos de 40 plantas por repetición, y se muestrearon cuatro plantas por repetición para determinar las variables de estudio.

El periodo de cosecha inició el 10 de noviembre del 2002 y concluyó el 20 de abril del 2003 (26 semanas), en el cual se evaluó: rendimiento total, rendimiento comercial, número y peso de frutos, y clasificación de frutos por su diámetro [chicos $(<6 \mathrm{~cm})$, medianos $(6.0$ a 7.0 $\mathrm{cm})$, grandes $(7.2$ a $8.0 \mathrm{~cm})$ y extra grandes $(>8.0 \mathrm{~cm})$, y frutos sin calidad comercial]. Se determinaron las concentraciones nutrimentales en muestras compuestas de hojas recientemente maduras y totalmente expandidas y extracto celular de peciolo, a los 17, 31, 45, 59, 73, 101, $118,151,169,195$ ddt. Las muestras de tejido vegetal se secaron a $70{ }^{\circ} \mathrm{C}$ por $48 \mathrm{~h}$ en una estufa con circulación forzada de aire y luego fueron molidas para su homogenización (Alcántar y Sandoval, 1999). La determinación de $\mathrm{N}$-total se hizo con la técnica de digestión semimicroKjeldahl (Horneck y Miller, 1998). La solubilización de $\mathrm{K}^{+}, \mathrm{Ca}^{2+}, \mathrm{Mg}^{2+}$ y micronutrimentos se realizó por digestión húmeda (Jones et al., 1992), y su determinación por absorción atómica (Thermo Serie M); P, S y B se determinaron mediante espectrofotometría de emisión por plasma (ICP-AES, PerkinElmer 3000 SCR) (Alcántar y Sandoval, 1999). A las muestras de extracto celular de peciolo se les determinó $\mathrm{N}-\mathrm{NO}_{3}$ mediante colorimetría, por el método del ácido acetil-salicílico, y $\mathrm{P}$ mediante ICP-AES, mientras que $\mathrm{K}$, Ca y $\mathrm{Mg}$ fueron determinados directamente mediante absorción atómica (Alcántar y Sandoval, 1999). Se determinó la eficiencia de uso del agua y fertilizantes en ambos medios de cultivo, mediante los cocientes: $\mathrm{kg}$ de fruta producida $/ \mathrm{m}^{3}$ de agua aplicada $\mathrm{y}$ $\mathrm{kg}$ de nutrimento aplicado/tonelada de fruta comercial producida. Las variables fueron sujetas a un análisis de varianza (ANOVA) y pruebas de comparación de medias por diferencia mínima significativa $(\alpha=0.05)$ con el paquete estadístico SAS V8.2 (SAS Institute, 1999).

\section{RESULTADOS Y DISCUSIÓN}

\section{Rendimiento comercial y tamaño de fruto}

La cosecha de frutos se llevó acabo durante $187 \mathrm{~d}$. El rendimiento comercial del tomate (Cuadro 2) no presentó diferencia significativa entre ambos sustratos, y osciló entre 30.4 y $34.1 \mathrm{~kg} \mathrm{~m}^{-2}$. Hao y Papadoupulos (2002) obtuvieron rendimientos de $33 \mathrm{~kg} \mathrm{~m}^{-2}$ al utilizar lana de roca como sustrato, que es similar a los obtenidos en este estudio. Estos resultados también concuerdan con Abad y Noguera (2000) quienes concluyeron que existen numerosos materiales que pueden ser utilizados exitosamente como sustratos en la producción hortícola, siempre y cuando su manejo (contenedor, riego y fertilización) esté adaptado a los requerimientos del medio y de la planta. El rendimiento comercial promedio por semana, para todo el ciclo, en los tratamiento de suelo y tezontles fue $1.31 \mathrm{y}$ $1.21 \mathrm{~kg} \mathrm{~m}^{-2}$ semanal (datos no mostrados), respectivamente; este rendimiento permite hacer comparaciones en diferentes condiciones y sistemas de producción.

Los frutos chicos $(\mathrm{Ch})$ y medianos (Med) presentaron diferencias significativas entre tratamientos (Cuadro 2). Aunque el tratamiento de suelo obtuvo el porcentaje más alto de frutos extra grandes (EG) y el menor en tamaños medianos (Med), no hubo diferencias significativas con 
los tratamientos de tezontle. Se registró la pudrición apical del fruto en todos los tratamientos en los meses de marzo y abril, que se atribuye a la alta temperatura y déficit hídricos, tanto en el suelo como en tezontle; en promedio, la pudrición afectó en $1.7 \%$ a los tratamientos en tezontle y en $2.1 \%$ al de suelo.

El peso promedio de fruto comercial para el tratamiento suelo fue de 77 (Ch), 133 (Med), 180 (Gde) y 237 (Ext. Gde). Para los tezontles fueron $68(\mathrm{Ch}), 120$ (Med), 156 (Gde) y 236 (Ext. Gde). Al respecto, los frutos de 180 a $230 \mathrm{~g}$ son los de mayor demanda y alcanzan mejor precio de venta en el mercado estadounidense.

\section{Concentración nutrimental en tejido vegetal y extracto celular de peciolo}

En la etapa vegetativa (17 a $45 \mathrm{ddt}$ ) no hubo diferencia significativa en la concentración de nitrógeno en tejido vegetal (Cuadro 3), mientras que en la etapa reproductiva (59 a $101 \mathrm{ddt}$ ) se registró la mayor concentración de $\mathrm{N}$ en suelo (3.85 \%) que en tezontle $(3.5,3.41$ y $3.37 \%$, respectivamente); a la cosecha (120 a 195 ddt), sólo en tezontle rojo la concentración de $\mathrm{N}$ fue significativamente menor (3.22 \%) que en suelo (3.69\%). En todos los tra- tamientos las concentraciones de $\mathrm{N}$ en tejido vegetal coinciden con los datos publicados por Sarro et al. (1986) de 3.4 a $4.4 \%$, por Jones et al. (1992) de 3.5 a $5 \%$, y por Castellanos (2004) de 3.3 a $4.5 \%$.

El contenido de $\mathrm{P}$ presentó diferencia significativa entre sustratos en las etapas vegetativa y reproductiva (Cuadro 3). Según Castellanos (2004), el nivel de suficiencia para fósforo total en tejido vegetal de tomate varía de 0.5 a $0.8 \%$ desde el trasplante hasta los $60 \mathrm{ddt}$; posteriormente puede variar de 0.35 a $0.7 \%$ hasta el final del ciclo del cultivo. La concentración de $\mathrm{K}$ fue significativamente mayor en suelo en la etapa vegetativa, lo que indica que las plantas jóvenes pueden asimilar más $\mathrm{K}$ en suelo que en la solución nutritiva del sustrato; en la etapa reproductiva los tratamientos evaluados no mostraron diferencias. Sin embargo, en la cosecha la concentración de K fue mayor en los tratamientos de sustrato que en el suelo. Un buen suministro de $\mathrm{K}$ en la etapa de mayor demanda (cosecha) permite obtener alta productividad y calidad de fruto (Dorais et al., 2001). El rango de suficiencia de $\mathrm{K}$ para esta etapa de cultivo, según Jones et al. (1992), es de 3.5 a $6.0 \%$, valores que concuerdan con las concentraciones aquí obtenidas.

Cuadro 2. Rendimiento y distribución por tamaños de tomate cv. 'Gironda' crecido en suelo y tezontle, en invernadero.

\begin{tabular}{|c|c|c|c|c|c|c|c|}
\hline \multirow[b]{2}{*}{ Tratamiento } & \multirow{2}{*}{$\begin{array}{c}\text { Rendimiento } \\
\text { total } \\
\left(\mathrm{kg} \mathrm{m}^{-2}\right)\end{array}$} & \multicolumn{5}{|c|}{ Fruto comercial } & \multirow{2}{*}{$\begin{array}{c}\text { Fruto no } \\
\text { comercial } \\
(\%)^{\Phi} \\
\text { Deformes }\end{array}$} \\
\hline & & $\begin{array}{l}\text { Rendimiento } \\
\left(\mathrm{kg} \mathrm{m}^{-2}\right)\end{array}$ & $\begin{array}{l}\text { Chico } \\
(\%)^{Y}\end{array}$ & $\begin{array}{r}\text { Mediano } \\
(\%)\end{array}$ & $\begin{array}{r}\text { Grande } \\
(\%)\end{array}$ & $\begin{array}{c}\text { Extra grande } \\
(\%)\end{array}$ & \\
\hline Suelo & $35.5 \mathrm{a}$ & $34.1 \mathrm{a}$ & $1.5 \mathrm{a}$ & $15.1 \mathrm{~b}$ & $61.1 \mathrm{a}$ & $22.7 \mathrm{a}$ & $1.9 \mathrm{a}$ \\
\hline${ }^{\dagger} \mathrm{TN}$ 25/75 & $31.3 \mathrm{~b}$ & $30.4 \mathrm{a}$ & $0.6 \mathrm{~b}$ & $25.5 \mathrm{a}$ & $59.8 \mathrm{a}$ & $14.2 \mathrm{a}$ & $1.1 \mathrm{a}$ \\
\hline TN $75 / 25$ & $34.1 \mathrm{ab}$ & $32.6 \mathrm{a}$ & $0.5 \mathrm{~b}$ & $22.4 \mathrm{ab}$ & $62.8 \mathrm{a}$ & $14.3 \mathrm{a}$ & $2.9 \mathrm{a}$ \\
\hline TR & $32.3 \mathrm{ab}$ & $31.2 \mathrm{a}$ & $0.8 \mathrm{ab}$ & $20.3 \mathrm{ab}$ & $64.2 \mathrm{a}$ & $14.8 \mathrm{a}$ & $1.7 \mathrm{a}$ \\
\hline DMS & 4.0 & ns & 0.8 & 10.7 & ns & ns & ns \\
\hline
\end{tabular}

${ }^{\top}$ Medias con la misma letra por columna son estadísticamente iguales (DMS, 0.05). ${ }^{\dagger} \mathrm{T}=$ Tezontle; $\mathrm{N}=$ Negro; $\mathrm{R}=$ Rojo. PAF $=$ Pudrición apical del fruto; chico $=70 \mathrm{~g}$; mediano $=123 \mathrm{~g}$; grande $=162 \mathrm{~g}$; Extra grande $=236 \mathrm{~g}$; ${ }^{{ }}$Peso de frutos como el porcentaje del rendimiento comercial. ${ }^{\Phi}$ Peso de frutos como el porcentaje del rendimiento total; ns = No significativo.

Cuadro 3. Concentración mineral en la hoja (en \% del peso seco) a lo largo del ciclo de producción de tomate cv. 'Gironda' en suelo y tezontle.

\begin{tabular}{|c|c|c|c|c|c|c|}
\hline Etapa Fenológica & Tratamiento & $\mathrm{N}$ & $\mathrm{P}$ & $\mathrm{K}$ & $\mathrm{Ca}$ & $\mathrm{Mg}$ \\
\hline Vegetativa & Suelo & $4.28 \mathrm{a}^{\ddagger}$ & $0.77 \mathrm{a}$ & $5.03 \mathrm{a}$ & $1.93 \mathrm{~b}$ & $0.54 \mathrm{~b}$ \\
\hline \multirow[t]{4}{*}{$(17-45 \mathrm{ddt})$} & ${ }^{\dagger} \mathrm{TN}$ 25/75 & $4.08 \mathrm{a}$ & $0.65 \mathrm{bc}$ & $4.53 \mathrm{~b}$ & $2.29 \mathrm{ab}$ & $0.63 \mathrm{a}$ \\
\hline & TN $75 / 25$ & $3.96 \mathrm{a}$ & $0.69 \mathrm{~b}$ & $4.22 \mathrm{~b}$ & $2.44 \mathrm{a}$ & $0.60 \mathrm{ab}$ \\
\hline & TR & $3.96 \mathrm{a}$ & $0.62 \mathrm{c}$ & $4.48 \mathrm{~b}$ & $2.43 \mathrm{a}$ & $0.54 \mathrm{~b}$ \\
\hline & DMS & ns & 0.05 & 0.48 & 0.41 & 0.07 \\
\hline Reproductiva & Suelo & $3.85 \mathrm{a}$ & $0.43 \mathrm{a}$ & $3.33 \mathrm{a}$ & $3.28 \mathrm{a}$ & $0.44 \mathrm{a}$ \\
\hline \multirow[t]{4}{*}{ (59 a $101 \mathrm{ddt})$} & TN 25/75 & $3.50 \mathrm{~b}$ & $0.62 \mathrm{~b}$ & $3.64 \mathrm{a}$ & $2.98 \mathrm{a}$ & $0.50 \mathrm{a}$ \\
\hline & TN $75 / 25$ & $3.41 \mathrm{~b}$ & $0.54 \mathrm{ab}$ & $3.70 \mathrm{a}$ & $2.99 \mathrm{a}$ & $0.50 \mathrm{a}$ \\
\hline & $\mathrm{TR}$ & $3.37 \mathrm{~b}$ & $0.50 \mathrm{ab}$ & $3.54 \mathrm{a}$ & $3.51 \mathrm{a}$ & $0.47 \mathrm{a}$ \\
\hline & DMS & 0.24 & 0.11 & ns & ns & ns \\
\hline Cosecha & Suelo & $3.69 \mathrm{a}$ & $0.34 \mathrm{a}$ & $3.47 \mathrm{~b}$ & $3.53 \mathrm{a}$ & $0.45 \mathrm{a}$ \\
\hline \multirow[t]{4}{*}{ (120 a $195 \mathrm{ddt})$} & TN $25 / 75$ & $3.43 \mathrm{ab}$ & $0.47 \mathrm{a}$ & $3.63 \mathrm{a}$ & $2.92 \mathrm{~b}$ & $0.47 \mathrm{a}$ \\
\hline & TN $75 / 25$ & $3.35 \mathrm{ab}$ & $0.44 \mathrm{a}$ & $3.75 \mathrm{a}$ & $2.89 \mathrm{~b}$ & $0.49 \mathrm{a}$ \\
\hline & TR & $3.22 \mathrm{~b}$ & $0.39 \mathrm{a}$ & $3.58 \mathrm{a}$ & $2.84 \mathrm{~b}$ & $0.44 \mathrm{a}$ \\
\hline & DMS & 0.37 & ns & 0.25 & 0.53 & ns \\
\hline
\end{tabular}

${ }^{\top}$ Medias con la misma letra por columna dentro de cada etapa fenológica son estadísticamente iguales (DMS, 0.05). ${ }^{\dagger} \mathrm{T}=\mathrm{Tezontle} ; \mathrm{N}=\mathrm{Negro} ; \mathrm{R}=\mathrm{Rojo}$; $\mathrm{ns}=$ No significativo. 
El Ca tuvo diferencia significativa en las etapas vegetativa y de cosecha; en esta última fue mayor en suelo que en tezontle, posiblemente debido a que en etapas avanzadas de desarrollo el suelo fue capaz de acumular $\mathrm{Ca}$ en la fase de intercambio, lo que permitió una mayor disponibilidad para la planta. El Mg presentó diferencias entre tratamientos sólo en la etapa vegetativa, pero las concentraciones de $\mathrm{Mg}$ aquí obtenidas están todas dentro de los rangos de suficiencias generados por Castellanos (2004) de 0.40 a $0.70 \%$, por Jones et al. (1992) de 0.50 a 0.75 $\%$, y por Sarro et al. (1986) de 0.64 a $0.78 \%$.

Debido a que los tratamientos de tezontle (25/75, $75 / 25$ y rojo) fueron estadísticamente iguales, se presenta la concentración promedio en extracto celular de peciolo de los tres tratamientos de este sustrato (Cuadro 4). Las concentraciones entre los dos sustratos tendieron a ser semejantes, con excepción del $\mathrm{Ca}$ y $\mathrm{Mg}$, en correspondencia con los análisis en hoja. En general, al comparar los análisis de tejido vegetal y los de extracto celular de peciolo se observó que cuando en tejido vegetal disminuyó la concentración de cualquier nutrimento, éste se reflejó en el contenido nutrimental en el extracto celular. En la etapa reproductiva el contenido de $\mathrm{P}$ en el extracto fue significativamente mayor en sustrato que en suelo, debido a que la concentración de $\mathrm{P}$ en la solución nutritiva aplicada al sustrato fue muy superior a la de la solución nutritiva que recibió el suelo. Sin embargo, dado que el rendimiento de fruto en el tratamiento de suelo no fue menor que en el sustrato, es de suponer que la concentración de P de sólo $200 \mathrm{mg} \mathrm{L}^{-1}$ en la etapa reproductiva haya sido suficiente para satisfacer la demanda del cultivo. Por otro lado, la concentración de Ca fue significativamente mayor en suelo que en sustrato en las dos últimas etapas de desarrollo, lo que sugiere que la dosis aplicada al suelo se puede reducir sin afectar el abastecimiento de este nutrimento.
Los niveles de suficiencia para $\mathrm{N}^{-\mathrm{NO}_{3}}{ }^{-}$en extracto celular de peciolo reportados en la literatura son contradictorios. Por ejemplo, en la Universidad de Florida se estableció un intervalo de 1200 a $700 \mathrm{mg} \mathrm{L}^{-1}$ (Hochmuth, 1994); Coltman (1988) lo ubicó de 1200 a $900 \mathrm{mg} \mathrm{L}^{-1}$, y Castellanos (2004) de 800 a $500 \mathrm{mg} \mathrm{L}^{-1}$, en la cosecha del tomate. Las concentraciones de $\mathrm{K}, \mathrm{P}, \mathrm{Ca}$ y $\mathrm{Mg}$ en el extracto celular estuvieron dentro de las concentraciones de suficiencia generadas por Sarro et al. (1986), Hochmutch (1994), Vega y Raya-De la Cruz (2000) y Castellanos (2004).

\section{Volumen de riego y eficiencia de uso del agua}

El manejo del riego es importante para conseguir altos rendimientos y alta calidad del producto. La cantidad de agua total aplicada a los tratamientos de tezontle fluctuó de 1055 a $1152 \mathrm{~L} \mathrm{~m}^{-2}$, con un drenaje de 31 a $38 \%$ del riego total aplicado (Cuadro 5). El agua consumida (volumen suministrado menos drenaje) por el cultivo fue de 675,706 y $790 \mathrm{~L} \mathrm{~m}^{-2}$ en los tratamientos de tezontle negro $(25 / 75)$, tezontle negro (75/25) y tezontle rojo. Estos datos concuerdan con los reportados por Hao y Papadoupulos (2002) quienes cultivaron tomate en lana de roca a solución perdida, y en un ciclo de 190 d determinaron que el cultivo consumió $652 \mathrm{~L} \mathrm{~m}^{-2}$ de agua, con rendimiento de $33.3 \mathrm{~kg} \mathrm{~m}^{-2}$, similar al presente trabajo. Papadopoulos (1991) recomienda aplicaciones extras de agua y nutrimentos ( 20 a $35 \%)$ en cultivos en sustrato para prevenir la acumulación de sales y desbalance nutrimental en la rizosfera. El tratamiento de suelo recibió $801 \mathrm{~L} \mathrm{~m}^{-2}$ en total que representó un ahorro de 24 a $30 \%$ de agua en relación con la suministrada a los tratamientos de tezontle, ahorro que es importante y una buena razón para usar suelo en la producción.

Cuadro 4. Concentración nutrimental en extracto celular de peciolo (en $\mathrm{mg} \mathrm{L}^{-1}$ ) por etapa fenológica de tomate cv. 'Gironda' crecido en suelo y tezontle.

\begin{tabular}{|c|c|c|c|c|c|c|}
\hline $\begin{array}{l}\text { Etapa } \\
\text { Fenológica }\end{array}$ & Tratamiento & $\mathrm{N}-\mathrm{NO}_{3}$ & $\mathrm{P}$ & K & $\mathrm{Ca}$ & $\mathrm{Mg}$ \\
\hline Vegetativa & Suelo & $787 \mathrm{a}^{\ddagger}$ & $366 \mathrm{a}$ & $3587 \mathrm{a}$ & $339 a$ & $260 \mathrm{~b}$ \\
\hline (17 a $45 \mathrm{ddt})$ & DMS ${ }^{\Uparrow}$ & ns & ns & ns & ns & 60 \\
\hline & Suelo & $595 \mathrm{a}$ & $200 \mathrm{~b}$ & $3912 \mathrm{a}$ & $453 \mathrm{a}$ & $289 a$ \\
\hline $\begin{array}{l}\text { (59 a } 101 \text { ddt) }\end{array}$ & Tezontle & $526 \mathrm{a}$ & $437 \mathrm{a}$ & $4357 \mathrm{a}$ & $260 \mathrm{~b}$ & $250 a$ \\
\hline \multirow{2}{*}{$\begin{array}{l}\text { Cosecha } \\
\text { (120 a } 195 \mathrm{ddt})\end{array}$} & Tezontle & $588 \mathrm{a}$ & $350 \mathrm{a}$ & $4280 \mathrm{a}$ & $352 \mathrm{~b}$ & $287 a$ \\
\hline & DMS ${ }^{\Uparrow}$ & ns & ns & ns & 74 & ns \\
\hline
\end{tabular}

${ }^{\top}$ Medias con la misma letra por columna dentro de cada etapa fenológica son estadísticamente iguales (DMS, 0.05). ns = No significativo. 
Cuadro 5. Suministro de riego total y por día, pérdidas por drenaje, consumo y eficiencia en el uso de agua en tomate cv 'Gironda' crecido en suelo y tezontle.

\begin{tabular}{|c|c|c|c|c|c|c|}
\hline \multirow{2}{*}{ Tratamiento } & \multirow{2}{*}{$\begin{array}{l}\text { Riego total } \\
\quad\left(\mathrm{L} \mathrm{m}^{-2}\right)\end{array}$} & Riego promedio & Drenaje & \multirow{2}{*}{$\begin{array}{c}\text { Drenaje } \\
(\%)\end{array}$} & \multirow{2}{*}{$\begin{array}{l}\text { Consumo de agua }{ }^{\dagger \dagger} \\
\qquad\left(\mathrm{L} \mathrm{m}^{-2} \mathrm{~d}^{-1}\right)\end{array}$} & \multirow{2}{*}{$\begin{array}{c}\mathrm{EUA}^{\mathrm{Y}} \\
\left(\mathrm{kg} \mathrm{m}^{-3}\right)\end{array}$} \\
\hline & & \multicolumn{2}{|c|}{$\left(\mathrm{L} \mathrm{m}^{-2} \mathrm{~d}^{-1}\right)$} & & & \\
\hline Suelo & 801 & 3.2 & --- & --- & 3.2 & $43 \mathrm{a}^{\pi}$ \\
\hline${ }^{\dagger} \mathrm{TN}$ 25/75 & 1055 & 5.0 & 1.8 & 36 & 3.2 & $29 \mathrm{~b}$ \\
\hline TN $75 / 25$ & 1138 & 5.4 & 2.1 & 38 & 3.4 & $29 \mathrm{~b}$ \\
\hline TR & 1151 & 5.5 & 1.7 & 31 & 3.8 & $27 \mathrm{~b}$ \\
\hline DMS & & & & & & 4.7 \\
\hline
\end{tabular}

${ }^{\top}$ Medias con la misma letra por columna son estadísticamente iguales (DMS, 0.05). ${ }^{\dagger} \mathrm{T}=$ Tezontle; $\mathrm{N}=\mathrm{Negro}$; $\mathrm{R}=$ Rojo. ${ }^{\dagger \dagger}$ Consumo de agua $=$ Volumen de riego - volumen de drenaje. ${ }^{\mathrm{V}} \mathrm{EUA}=$ Eficiencia en el uso de agua.

Dos aspectos importantes acerca de la eficiencia del suministro de riego son el consumo del agua y la eficiencia en el uso del agua (EUA). El tratamiento suelo fue el que presentó mayor EUA (43 $\mathrm{kg}$ de fruta $\mathrm{m}^{-3}$ de agua), en comparación con los tratamientos de tezontle que oscilaron entre 27 y $29 \mathrm{~kg}$ de tomate $\mathrm{m}^{-3}$ de agua (Cuadro 5). La mayor EUA del suelo se debió a la baja fracción de drenaje, la cual se estima que apenas llegó a $5 \%$, mientras que en los tratamientos de tezontle los valores de drenaje oscilaron entre 31 y $38 \%$. Los tratamientos de tezontle, tanto rojo como negro, presentan valores de EUA de 27 a $29 \mathrm{~kg} \mathrm{~m}^{-3}$, similares a los reportados por Lorenzo et al. (1993) de $29 \mathrm{~kg}$ de tomate $\mathrm{m}^{-3}$ de agua, en sustrato de perlita con una solución nutritiva de $3.0 \mathrm{dS} \mathrm{m}^{-1}$ de conductividad eléctrica. Según Stanghellini (2003; Com. personal) ${ }^{1}$, en invernaderos de cristal sin calefacción en Israel, se obtienen $33 \mathrm{~kg}$ de tomate $\mathrm{m}^{-3}$ de agua en sustrato, mientras que en Almería, España en invernaderos semi-automatizados o de tecnología intermedia bajo condiciones de suelo se alcanza un valor de $37 \mathrm{~kg} \mathrm{~m}^{-3}$ de agua.

\section{Suministro y uso de nutrimentos en la producción}

Los mayores niveles de suministros de nutrimentos fueron en los sustratos de tezontle, en comparación con el suelo (Cuadro 6). Esto se debe a que en el cultivo sin suelo las pérdidas de nutrimentos en el drenaje pueden sobrepasar de $50 \%$ de la cantidad aplicada, como ocurrió con N y K. Hao y Papadopoulos (2002) registraron pérdidas de nutrimentos por drenaje de 48, 35, 42, 65 y $54 \%$ de $\mathrm{N}, \mathrm{P}_{2} \mathrm{O}_{5}, \mathrm{~K}_{2} \mathrm{O}$, Ca y $\mathrm{Mg}$, respectivamente, para tomate cultivado en lana de roca en un sistema abierto, con un rendimiento comercial de $33.3 \mathrm{~kg} \mathrm{~m}^{-2}$, similar al aquí obtenido. Es notable la mayor eficiencia del sistema suelo en comparación con el cultivo sin suelo. Quizás por ello 80 $\%$ de los productores en España han usado este sistema de producción desde hace muchos años (Castilla, 2005). Algunos autores se muestran reacios a considerar el suelo

\footnotetext{
${ }^{1}$ Stanghellini C (2003) El agua de riego: su uso, eficiencia y economía. In: Curso Superior de Especialización sobre Mejoras de la Eficiencia en el Uso del Agua en Cultivos Protegidos. F.I.A.P.A. CAJAMAR. pp:2536.
}

como medio de cultivo por razones fitosanitarias; sin embargo, con el reciente surgimiento del injerto es factible enfrentar las enfermedades provenientes del suelo sin mayor problema, aun en el caso de monocultivo (CamachoFerre y Tello-Marqina, 2006). Si esta tecnología del injerto se complementa con solarización y manejo de materia orgánica, el cultivo en suelo acabará por imponerse en muchas regiones de México, debido a su mayor eficiencia de uso de fertilizantes y agua y a la menor vulnerabilidad del sistema de producción a los fallos de manejo, como se ha demostrado en España (Camacho-Ferre y TelloMarqina, 2006).

El consumo de $\mathrm{N}, \mathrm{P}_{2} \mathrm{O}_{5}$ y $\mathrm{K}_{2} \mathrm{O}$ registrados en los tratamientos de tezontle, concuerdan con los obtenidos por White (1993) de 790, 389, $2053 \mathrm{~kg} \mathrm{ha}^{-1}$ de N, $\mathrm{P}_{2} \mathrm{O}_{5}, \mathrm{~K}_{2} \mathrm{O}$ respectivamente, en tomate cultivado con la técnica de película de nutrientes (NFT) en un ciclo de $238 \mathrm{~d}$; este mismo autor estimó para $\mathrm{Ca}$ y $\mathrm{Mg}$ valores de 606 y 112 $\mathrm{kg} \mathrm{ha}^{-1}$ respectivamente, inferiores a los aquí obtenidos.

El suelo superó a los tezontles en eficiencia de uso de nutrimentos (Cuadro 7). Los tratamientos de tezontle mantuvieron un promedio de 5.6, 1.4, 11.7, 7.0 y $2.3 \mathrm{~kg}$ de $\mathrm{N}, \mathrm{P}_{2} \mathrm{O}_{5}, \mathrm{~K}_{2} \mathrm{O}$, Ca y $\mathrm{Mg}$, respectivamente, por tonelada de fruto comercial, semejante a los encontrados por Hao y Papadoupulos (2002) de 6.7, 2.8, 10.7, 5.4 y 1.3 $\mathrm{kg} \mathrm{t}^{-1}$ de fruto comercial, con una pérdida por drenaje de

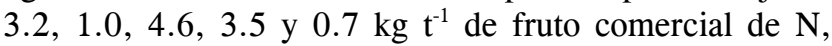
$\mathrm{P}_{2} \mathrm{O}_{5}, \mathrm{~K}_{2} \mathrm{O}$, Ca y $\mathrm{Mg}$, respectivamente, en un cultivo de tomate en lana de roca a solución perdida, y con un rendimiento comercial de $33.3 \mathrm{~kg} \mathrm{~m}^{-2}$. En el presente estudio, los valores en suelo fueron la mitad de los registrados para tezontle en todos los casos. Estos resultados ponen en evidencia el potencial de ahorro en nutrimentos para la producción de tomate que se puede conseguir mediante fertirrigación en suelo, comparado con el sistema de cultivo en tezontle a solución perdida. 
Cuadro 6. Suministro, drenado y absorción de nutrimentos $\left(\mathrm{kg} \mathrm{ha}^{-1}\right)$ en el tomate cv "Gironda" en invernadero crecido en suelo y tezontle.

\begin{tabular}{|c|c|c|c|c|c|}
\hline Tratamiento & $\mathrm{N}$ & $\mathrm{P}_{2} \mathrm{O}_{5}$ & $\mathrm{~K}_{2} \mathrm{O}$ & $\mathrm{Ca}$ & $\mathrm{Mg}$ \\
\hline `Suministro en suelo & 920 & 342 & 1520 & 1050 & 290 \\
\hline \multicolumn{6}{|l|}{ Suministro en tezontle } \\
\hline 'TN 25/75 & 1661 & 427 & 3430 & 2055 & 705 \\
\hline TR & 1798 & 469 & 3794 & 2236 & 739 \\
\hline \multicolumn{6}{|l|}{ Drenado en el tezontle } \\
\hline TN 25/75 & 961 & 33 & 1436 & 1003 & 426 \\
\hline $\begin{array}{l}\% \text { medio de pérdidas } \\
\text { respecto al total suministrado }\end{array}$ & 56 & 6 & 42 & 48 & 60 \\
\hline \multicolumn{6}{|l|}{ Consumo de nutrimentos } \\
\hline TN $25 / 75$ & 700 & 393 & 1994 & 1052 & 279 \\
\hline TN $75 / 25$ & 722 & 433 & 2072 & 1090 & 293 \\
\hline $\mathrm{TR}$ & 879 & 447 & 2237 & 1244 & 308 \\
\hline
\end{tabular}

${ }^{\complement}$ Total acumulado de nutrimentos suministrados en la solución nutritiva. ${ }^{\dagger} \mathrm{T}=$ Tezontle; $\mathrm{N}=$ Negro; $\mathrm{R}=$ Rojo.

Cuadro 7. Eficiencia de uso de los nutrimentos (en kg por tonelada de fruto comercial), con base en las cantidades totales aplicadas.

\begin{tabular}{|c|c|c|c|c|c|}
\hline Tratamiento & $\mathrm{N}$ & $\mathrm{P}_{2} \mathrm{O}_{5}$ & $\mathrm{~K}_{2} \mathrm{O}$ & $\mathrm{Ca}$ & $\mathrm{Mg}$ \\
\hline TN $75 / 25$ & 5.5 & 1.4 & 11.3 & 6.8 & 2.3 \\
\hline
\end{tabular}

${ }^{\dagger} \mathrm{T}=$ Tezontle; $\mathrm{N}=$ Negro; $\mathrm{R}=$ Rojo.

El coeficiente de consumo para el sistema de producción de tezontle se mantuvo de 2.2-2.8, 1.3-1.9, 4.5-7.2, $3.1-4.0$ y $0.9-1.9 \mathrm{~kg} \mathrm{t}^{-1}$ de fruto comercial de $\mathrm{N}, \mathrm{P}_{2} \mathrm{O}_{5}$, $\mathrm{K}_{2} \mathrm{O}$, Ca y $\mathrm{Mg}$, respectivamente, valores semejantes a los encontrados por Hao y Papadoupulos (2002) quienes reportaron coeficientes de consumo de nutrimentos de 3.5 , $1.8,6.1,2.0$ y $0.6 \mathrm{~kg} \mathrm{t}^{-1}$ de fruto comercial para $\mathrm{N}, \mathrm{P}_{2} \mathrm{O}_{5}$, $\mathrm{K}_{2} \mathrm{O}$, Ca y $\mathrm{Mg}$.

\section{CONCLUSIONES}

El potencial de ahorro en agua y fertilizante que se puede tener en condiciones de cultivo en suelo, puede ser del orden de $50 \%$ en fertilizante y de $70 \%$ en agua, en comparación con el sistema en sustrato de tezontle, aunque ambos sistemas produjeron el mismo rendimiento de fruto. Sin embargo, en condiciones de suelo seriá necesario usar la técnica de injerto mediante el uso de portainjertos resistentes a patógenos de suelo para asegurar el éxito de este sistema en el largo plazo.

\section{BIBLIOGRAFÍA}

Abad M, P Noguera (2000) Los sustratos en los cultivos sin suelo. In: Manual de Cultivo sin Suelo. M Urrestarazu (ed). 2a ed. Mundi-Prensa. Madrid, España. pp:137-185.

Alcántar G G, V M Sandoval (1999) Manual de Análisis Químico de Tejido Vegetal. Guía de Muestreo, Preparación Análisis e Interpretación. Publicación especial número 10 de la Sociedad Mexicana de la Ciencia del Suelo, A.C. Centro de Edafología. Colegio de Posgraduados. $156 \mathrm{p}$.
Alcántar G G, R M Villareal (1999) Tomato growth (Lycopersicon esculentum Mill.) and nutrient utilization in response to varying fertigation programs. Acta Hort. 481:385-389.

Andriolo J L, R A Grave, R H Bartz (2002) Effect of fertilizer level on tissue nitrogen concentration of greenhouse tomato plants grown in substrate culture. Acta Hort. 559:607-610.

Baca C G A, B S Alcalde, G A Martínez, L R James, I D Barrera (1991) Efecto de la solución nutritiva, riego, el sustrato y la densidad de siembra en tres cultivos hortícolas en la hidroponía al aire libre. II Melón y jitomate. Agrociencia 2:33-55.

Bastida A (1999) El Medio de Cultivo de las Plantas. Sustratos para Hidroponía y Producción de Plantas Ornamentales. Serie de publicaciones AGRIBOT No. 4 UACH. Preparatoria Agrícola, Chapingo, Mex. $72 \mathrm{p}$.

Camacho Ferre F, J C Tello Marquina (2006) Control de Patógenos Telúricos en Cultivos Hortícolas Intensivos. Ed. Agrotécnicas. Madrid, España. 160 p.

Castellanos J Z (2004) Manejo de la fertirrigación en suelo. In: Manual de Producción Hortícola en Invernadero. J Z Castellanos (ed). 2a ed. INTAGRI. México. pp:103-123.

Castellanos J Z, P Vargas Tapia (2003) El uso de sustratos en la horticultura bajo invernadero. In: Manual de Producción Hortícola en Invernadero. J Z Castellanos (ed). 2a ed. INTAGRI. México. pp:124-150.

Castilla N (2005) Invernaderos de Plástico, Tecnología y Manejo. Ed. Mundi-Prensa. Madrid, España. $462 \mathrm{p}$.

Coltman R R (1988) Yield of greenhouse tomatoes managed to maintain specific petiole sap nitrate levels. HortScience 23:148-151.

Coltman R R, S A Riede (1992) Monitoring the potassium status of greenhouse tomatoes using quick petiole sap test. HortScience 27:361-364.

Dorais M, A P Papadopulos, A Gosselin (2001) Greenhouse tomato fruit quality. Hort. Rev. 26:263-319.

Grijalva Contreras R, R Macias, M J Valenzuela, F Robles (2004) Productivity and fruit quality in tomato varieties under greenhouse conditions in the Northwest of Mexico. HortScience 39:745-897 
Hao X, A P Papadopoulos (2002) Growth, photosynthesis and productivity of greenhouse tomato cultivated in open o closed rockwool systems. Can. J. Plant Sci. 82:771-780.

Hochmuth G J (1994) Efficiency ranges for nitrate-nitrogen and potassium for vegetable petiole sap quick test. HortTechnology 4:218-222.

Horneck D A, R O Miller (1998) Determination of total nitrogen in plant tissue. In: Handbook of Reference Methods for Plant Analysis Tissue. Let Plants Speak. Y P Kalra (ed). Soil and Plant Analysis Council. CRC Press, Boca Ratón. pp:75-84.

Inden H, A Torres (2004) Comparison of four substrate on the growth and quality of tomatoes. Acta Hort. 644:205-210.

Jackson M L (1964) Análisis Químico de Suelos. J Beltrán O (trad). Barcelona, España. 185 p.

Jones J B Jr, B Wolf, H A Mills (1992) Plant Analysis Handbook: A Practical Sampling, Preparation, Analysis and Interpretation Guide. Micro Macro Publishing Inc. Athens, GA. 213 p.

Jones J B Jr (1999) Tomato plant culture. In: Plant Nutrition in the Field, Greenhouse and Home Garden. CRC Press LLC (ed). Boca Raton, FL. pp:51-72.

Lorenzo P, E Medrano, M L García (1993) Irrigation management in perlita. Acta Hort. 335:429-434.
Muñoz R J J (2003) La producción de hortalizas bajo invernadero en México. In: Manual de Producción Hortícola en Invernadero. J Z Castellanos, J J Muñoz R (eds). INTAGRI. México. pp: 14-16.

Papadopoulos A P (1991) Growing greenhouse tomatoes in soil and soilless media. Agriculture and Agri-Food Canada, Ottawa, ON. Publ. 1865/E. 79 p.

Sarro C M J, C L Cadahia, O J M Peñalosa (1986) Control de un cultivo hidropónico de tomate mediante correcciones periódicas de los nutrientes. In: Aplicaciones a los Invernaderos Comerciales. Anal. Edaf. Agrobiol. XLV, 5-6:729-738.

SAS Institute (1999) The SAS system for windows. Release 8.2. SAS Institute Cary, NC.

Vega L M, J L Raya-De la Cruz (2000) Cultivo en lana de roca. Parte I. In: Manual de Cultivos sin Suelo. M Urrestarazu (coord). Ed. Mundi-Prensa. Madrid. pp:481-515.

Villareal R M, E R García, E T Osuna, B A Armenta (2002) Efecto de dosis y fuente de nitrógeno en rendimiento y calidad de poscosecha de tomate en fertirriego. Terra 20:311-320.

White J R A (1993) Nutrient uptake by tomatoes grown in NFT. In: Proc. 8th Int. Congr. Soilless Culture. Int. Soc. Soilless Culture (ISOSC). Hunters Rest, South Africa. pp:483-496. 